\title{
Einfluss von Permanent Make-up auf die Meibomdrüsen und den Tränenfilm
}

\author{
Christoph Laufenböck (D)
}

Eingegangen: 10. Juni 2020 / Angenommen: 27. November 2020 / Online publiziert: 14. Dezember 2020

(c) Der/die Autor(en) 2020

\section{Zusammenfassung}

Hintergrund Ziel dieser Studie war es, Veränderungen hinsichtlich der Meibomdrüsen, der Augenoberfläche und des Tränenfilms durch Lidrandtätowierungen zu ermitteln.

Methodik Querschnittsstudie: 100 Augen mit Permanent-Make-up von 50 Patientinnen wurden $100 \mathrm{Au}$ gen ohne Permanent-Make-up von 50 Patientinnen gegenübergestellt. Die subjektive Symptomabfrage erfolgte mittels OSDI-Fragebogen und wurde mit den objektiven Testergebnissen - erhoben durch die klinische Untersuchung an der Spaltlampe sowie am Keratographen 5M (Oculus, Germany) - verglichen.

Resultate Es zeigte sich ein statistisch signifikanter Unterschied zwischen den beiden Gruppen hinsichtlich des OSD-Index, der NIK-BUT, der Meibomdrüsen und der LIPKO-Falten. Keinen Unterschied zeigte sich in der bulbären Injektion und der Tränenmeniskushöhe.

Schlussfolgerungen In der durchgeführten Studie zeigte sich, dass Permanent-Make-up an den Lidrändern $\mathrm{zu}$ anatomischen und funktionellen Veränderungen der Meibomdrüsen und des Tränenfilms führt. Diese Ergebnisse korrelieren auch stark mit einem subjektiven okulären Dyskomfort der Patientinnen.

Schlüsselwörter Permanent-Make-up · Augenlid · Tränenfilminstabilität · OSD · Meibomdrüsen

Dr. med. univ. C. Laufenböck, FEBO ( $₫)$

Augenordination, Färbergasse 13, 6850 Dornbirn, Österreich christoph.laufenboeck@gmx.at
Influence of eyelid tattooing on meibomian glands and tear film

\section{Summary}

Purpose To evaluate changes on the meibomian glands, the ocular surface and the tear film after eyelid tattooing.

Methods Cross-sectional study: 100 eyes with eyelid tattooing of 50 female patients were compared to 100 untreated eyes of 50 female patients. Symptoms were assessed using the OSD-Index. We used the biomicroscopy to estimate the lid parallel conjunctival folds. We used the Keratograph $5 \mathrm{M}^{\circledR}$ (Oculus, Germany) to figure out the NIK-BUT, the conjunctival injection, the anatomical condition of the meibomian glands and the tear meniscus height.

Results There was a statistically significant difference between tattooed and non-tattooed eyelids concerning the OSD-Index, the NIK-BUT, the meibomian glands and lid parallel conjunctival folds. There was no statistical significance concerning the nasal/ temporal conjunctival injection and the tear meniscus height.

Conclusion Eyelid tattooing increases tear film instability through meibomian gland disturbance und tear film instability and could aggravate signs and symptoms of ocular surface disease.

Keywords Eyelid tattooing · Eyelid tear film • Instability $\cdot$ OSD $\cdot$ Meibomian glands

\section{Einleitung}

Der präkorneale Tränenfilm ist eine sehr dünne Flüssigkeitsschicht über der Hornhautoberfläche. Seine Aufgaben beinhalten die Aufrechterhaltung einer glatten Brechungsfläche im Zusammenspiel von drei Schichten: die Muzin-Schicht hilft die Tränenflüssig- 
keit an der Augenoberfläche zu halten. Die wässrige Schicht, ein Gemisch auf Peptiden/Proteinen, Kochsalz- und Elektrolyten, bringt der Hornhaut Feuchtigkeit und Sauerstoff und spielt eine wichtige Rolle neben anderen zur Benetzung der der Augenoberfläche und die Lipidschicht - produziert von den Meibom-/ Moll- und Zeisdrüsen - sorgt für eine stabile Oberflächenspannung und verhindert die Evaporation [1, 2].

Die ocular surface disease (OSD) bezieht sich auf ein breites Spektrum von Erkrankungen der Augenoberfläche - bedingt entweder durch ein Mangel der Lipidschicht, des wässrigen Anteils, der Muzinschicht oder durch Mischformen, die zu Symptomen des trockenen Auges, einem Abfall der Sehleistung und somit zur Verminderung der Lebensqualität führen [3-10]. Die Meibomdrüsendysfunktion resultiert in einer qualitativen und quantitativen Veränderung der Lipidschicht mit konsekutiver Instabilität des Tränenfilms. Diagnostisch erhebbar kann der funktionelle Aspekt mittels der Tränenaufrisszeit (BUT) und des funktionellen Visus, der morphologische Aspekt mit der Meibographie, des Vorderabschnitts-OCT und der Interferometrie werden [11-16].

Das Permanent Make-up wird seit Jahren immer beliebter. Es zeigten sich bei histopathologischen Untersuchungen eine Persistenz des eingebrachten Pigments als freie Granula in der Epidermis und Dermis, die meisten in Makrophagen in der Dermis und im Bereich der Lidränder an der Oberfläche des OrbicularisMuskels. Als Nebenwirkungen können neben traumatischen Verletzungen während der kosmetischen Behandlung (dabei liegen auch Fallstudien vor, die eine akzidentielle Verletzung der Hornhaut demonstrieren) Allergien, Dislokation des Pigments sowohl oberflächlich als auch in tiefere Schichten, Entzündungen, Nekrosen und Granulome entstehen [17-25].

Technisch wird nahe dem äußeren Lidrand Pigment in die Haut mittels dafür geeigneter Pigmentiergeräte in hunderten Einzelstichen eingebracht. Allein aus der anatomischen Lokalisation liegt es nahe, dass möglicherweise die Meibomdrüsen bzw. deren Drüsenausgänge in Morphologie und Funktion gestört werden könnten. In dieser Studie versuchen wir einen denkbaren negativfunktionellen Effekt diesbezüglich im Vergleich zu einem nicht behandelten Probandinnenkollektiv nachzuweisen.

\section{Methodik}

100 weibliche Probandinnen wurden rekrutiert. Dabei hatten 100 Augen ein Permanent-Make-up an Unterund Oberlid seit mindestens 3 Jahren und 100 Augen waren unbehandelt. Es erfolgte neben der Erhebung des OSD-Index eine genaue Anamnese hinsichtlich des Zeitpunktes der kosmetischen Behandlung, der Krankengeschichte mit besonderer Beachtung eventueller Schilddrüsenpathologien, der Medikamenteneinnahme inklusive psychoaktiver Substanzen, des
Nikotin-/Alkoholkonsum sowie der täglichen Trinkmenge. Ausgeschlossen wurden Probandinnen wegen: florider Bindehaut-/Hornhautentzündung, Demodexbefall, lokaler Vormedikation, Glaukom, fazialer Erkrankungen (z. B. Rosazea), Lidfehlstellungen wie Ektropia, Tränenwegsstenosen und Zustände nach Gesichtstraumata.

Erhoben wurden mittels des Keratographen 5 der Firma Oculus die NIK-BUT, die Tränenmeniskushöhe, die bulbäre Rötung temporal und nasal, die LIPKOFalten sowie der Status der Meibomdrüsen (Meibographie, Einteilung nach der "Meiboscale by Dr. Pult“). Alle statistischen Analysen wurden durchgeführt mittels Excel Office 16 und IBM SPSS ${ }^{\circledR}$ Statistics. $P<0,05$ galt als signifikant.

Obwohl keine medizinische Intervention stattfand, wurde jede Probandin schriftlich und mündlich über die Studie informiert.

\section{Ergebnisse}

Das durchschnittliche Lebensalter der Probandinnen lag in der Tattoo-Gruppe bei 58 Jahren $( \pm 12)$ und in der unbehandelten Gruppe bei 55 Jahren $( \pm 10)$. Das Alter des Permanent-Make-ups betrug 9,3 Jahre $( \pm 4,0)$. Darüber hinaus konnten wir einen Anteil von Raucherinnen mit $23 \%$ und von Schilddrüsenpathologien mit $17 \%$ bestimmen.

Signifikante Unterschiede zugunsten der nicht tätowierten Gruppe konnten im Ergebnis des OSDIndex $(19,0 \pm 8,01$ vs. $10,8 \pm 3,56, p 0,0004$, Mann-Whitney $U$ Test) sowie in der Meibographie $(2,5 \pm 0,67$ vs. $1,6 \pm 0,68, p 0,0001)$, der NIK-BUT $(9,6 \pm 3,35$ vs. $10,7 \pm 3,95, p 0,0001)$ und der LIPKO-Falten $(2,3 \pm 0,50$ vs. $1,3 \pm 0,48, p 0,0004)$ dargestellt werden. Nicht signifikante Unterschiede fanden wir in der nasalen $(1,6 \pm 0,78$ vs. $1,5 \pm 0,52, p 0,22)$ und der temporalen Rötung $(1,3 \pm 0,53$ vs. $1,1 \pm 0,09, p 0,1)$ sowie in der Tränenmeniskushöhe $(0,4 \pm 0,14$ vs. $0,42 \pm 0,07$, $p$ 0,67). Einen Unterschied zwischen Raucherinnen/ Nichtraucherinnen bzw. zwischen Probandinnen oh-

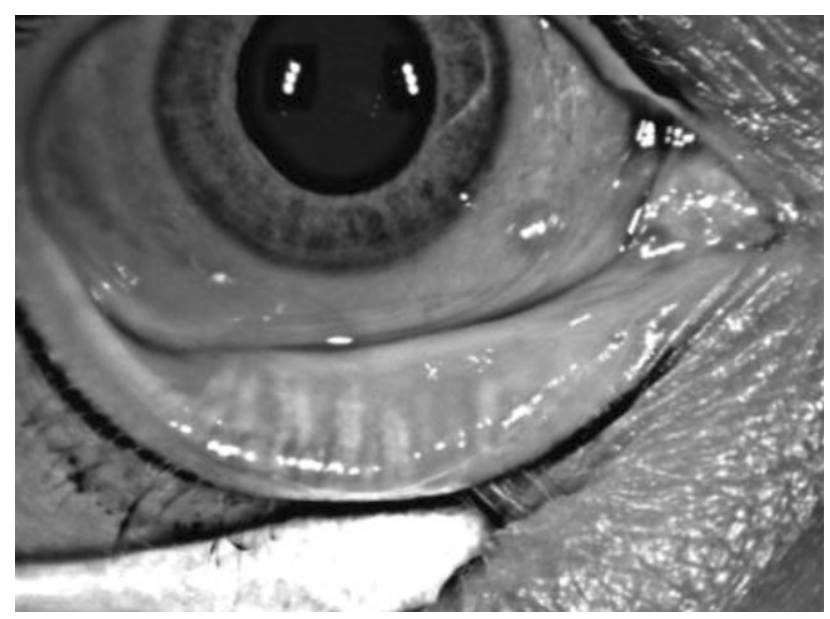

Abb. 1 Meibomdrüsenausfälle am Unterlid rechts 


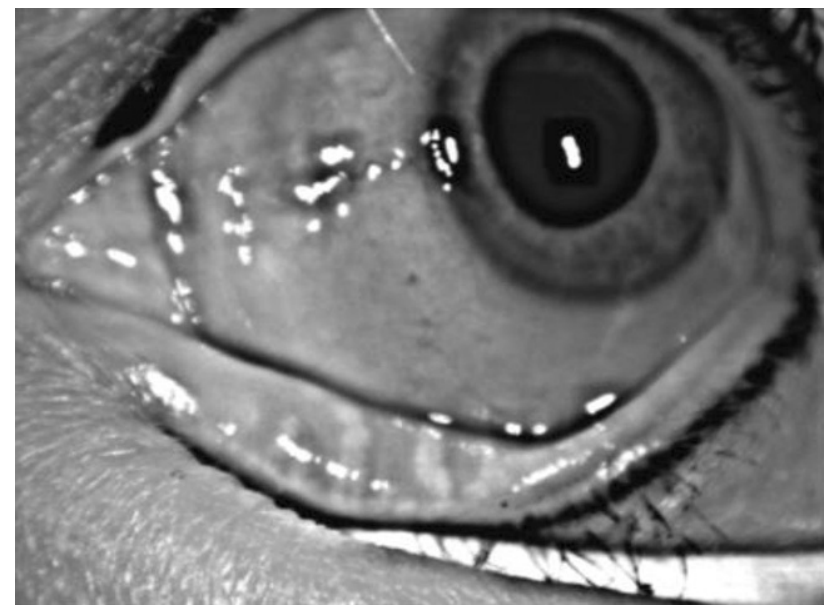

Abb. 2 Meibomdrüsenausfälle am Unterlid links
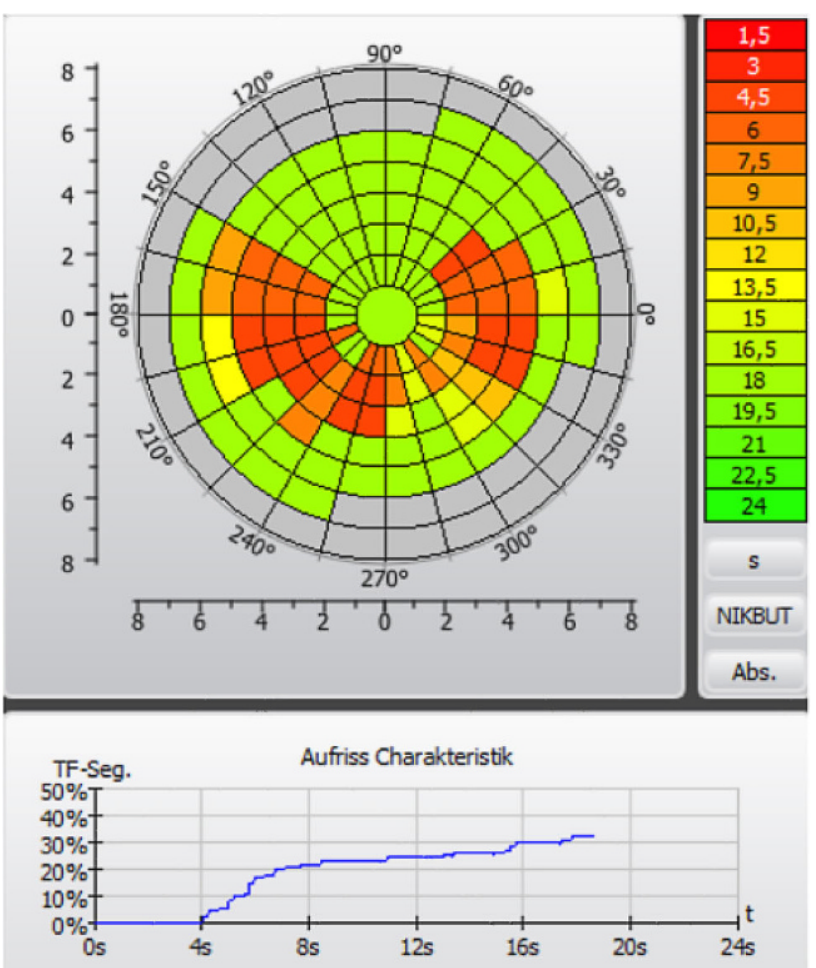

Aufriss (1.): $4.08 \mathrm{~s}$

Aufriss (Ø): $8.33 \mathrm{~s}$

Kassifikation: Grad 1

Haftungsausschluss: Dieses Programm erstellt keine medizinische Diagnose und dient nicht dazu, mögliche Therapien, ohne weitere professionelle Untersuchung und weitere medizinische Befunde oder diagnostische Tests, vorzugeben.

Abb. 3 Stark verkürzte NIK-BUT bei mit Permanent Make-up vorbehandelten Lidrändern

ne oder mit Schilddrüsenpathologien konnten nicht nachgewiesen werden.
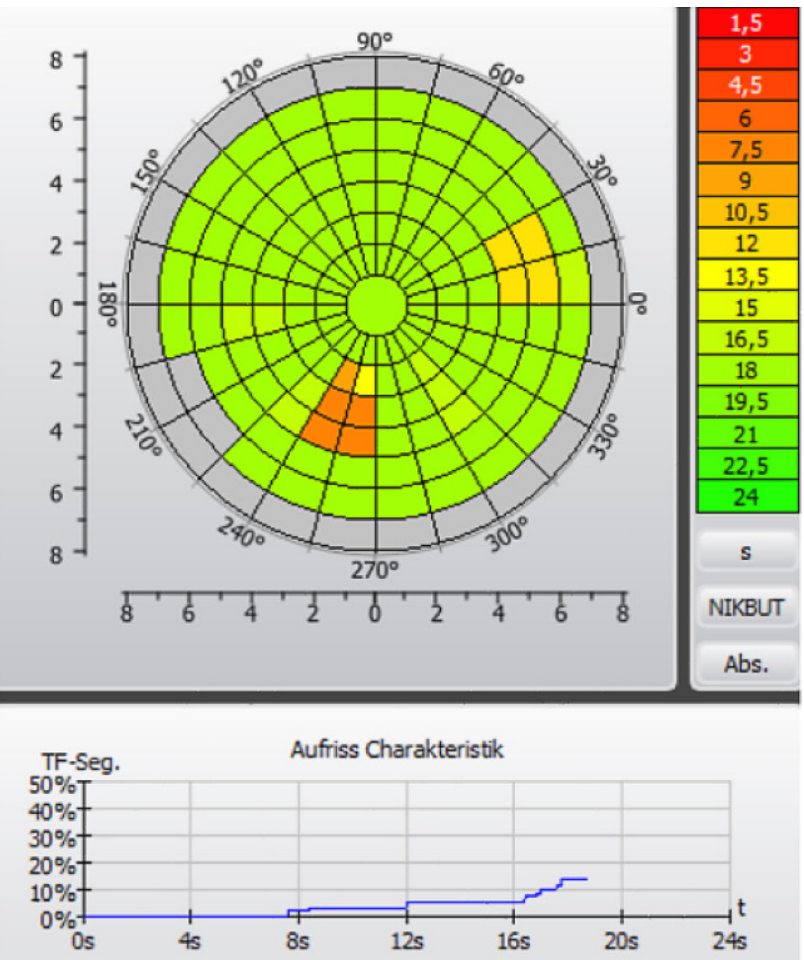

Aufriss (1.): $7.65 \mathrm{~s}$

Aufriss $(\varnothing): \quad 14.46 \mathrm{~s}$

Sassifikation: Level 0

Haftungsausschluss: Dieses Programm erstellt keine medizinische Diagnose und dient nicht dazu, mögliche Therapien, ohne weitere professionelle Untersuchung und weitere medizinische Befunde oder diagnostische Tests, vorzugeben.

Abb. 4 Annähernd normale NIK-BUT bei unbehandelten Lidrändern

\section{Diskussion}

Meibomdrüsen sind an den Augenlidern befindliche Talgdrüsen. Im Oberlid des Auges befinden sich etwa 30, im Unterlid etwa 20 tubuloalveolär verzweigte Drüsen. Dem Bau nach sind sie holokrine Talgdrüsen, d.h. der Talg (das Meibum) entsteht durch den Untergang ganzer Zellen des Drüsenepithels [26, 27]. Das lipidreiche Sekret nimmt nach jedem Lidschlag Kontakt mit der wässrigen Phase des Tränenfilms auf und verhindert eine Evaporation [28, 29].

In dieser Studie zeigten sich alle Parameter, die direkt oder indirekt auf eine Meibomdrüsendysfunktion hinweisen können, in der Tattoo-Gruppe signifikant schlechter als in der unbehandelten Gruppe. Sowohl der OSD-Index, die Meibographie (Abb. 1 und 2), die NIK-But (Abb. 3 und 4) und die LIPKO-Falten präsentierten sowohl subjektive als auch objektive Hinweise auf das Vorliegen eines Sicca-Syndroms bedingt durch eine Meibomdrüsendysfunktion und eine konsekutive Hyperevaporation nach Einbringen der Tattootinte am Lidrand. Die Tränenmeniskushöhe und die bulbäre Rötung zeigten keine signifikanten Unterschiede in den beiden Gruppen. Dies ist durchaus in Einklang zu 
bringen mit vorangegangenen dermatologischen Studien als auch ophthalmologischen Fallberichten, dass Tattoos nicht nur zu lokalen Infektionen, toxischen Prozessen und Allergien sondern auch zu einem Eindringen der Tinte in die Tiefe führen können [30-35]. Dies ist ein Vorgang, der häufig als „Ausbleichen“ verstanden wird und zu einer weiteren kosmetischen Anwendung führt. Auch eine direkte mechanische Verletzung der Meibomdrüsen bei zu großer Eindringtiefe ist im Prinzip vorstellbar [18]. Darüber hinaus sind die Inhaltsstoffe der applizierten Tinte nicht standardisiert und können verschiedenste Substanzen bis hin zu Arsen enthalten [32, 34-36].

Eine anzuschließende histologische Studie in der Zukunft zur Darstellung der genauen anatomischen Veränderungen der Meibomdrüsen nach kosmetischer Lidrandtätowierung wäre wünschenswert.

\section{Zusammenfassung}

Zusammenfassend kann festgehalten werden, dass in dieser vergleichenden Beobachtungsstudie ein instabilerer Tränenfilm in Augen mit Permanent-Make-up auftrat im Vergleich zu unbehandelten Lidrändern. Dies ist auf eine Meibomdrüsendysfunktion zurückzuführen und zeigt, dass Permanent-Make-up ein weiterer Risikofaktor für die Entwicklung des trockenen Auges darstellt.

\section{Einhaltung ethischer Richtlinien}

Interessenkonflikt C. Laufenböck gibt an, dass kein Interessenkonflikt besteht.

Ethische Standards Die Patientinnen gaben deren Einwilligung zur Teilnahme an der Studie und unterzeichneten die Patienteninformation.

Open Access Dieser Artikel wird unter der Creative Commons Namensnennung 4.0 International Lizenz veröffentlicht, welche die Nutzung, Vervielfältigung, Bearbeitung, Verbreitung und Wiedergabe in jeglichem Medium und Format erlaubt, sofern Sie den/die ursprünglichen Autor(en) und die Quelle ordnungsgemäß nennen, einen Link zur Creative Commons Lizenz beifügen und angeben, ob Änderungen vorgenommen wurden.

Die in diesem Artikel enthaltenen Bilder und sonstiges Drittmaterial unterliegen ebenfalls der genannten Creative Commons Lizenz, sofern sich aus der Abbildungslegende nichts anderes ergibt. Sofern das betreffende Material nicht unter der genannten Creative Commons Lizenz steht und die betreffende Handlung nicht nach gesetzlichen Vorschriften erlaubt ist, ist für die oben aufgeführten Weiterverwendungen des Materials die Einwilligung des jeweiligen Rechteinhabers einzuholen.

Weitere Details zur Lizenz entnehmen Sie bitte der Lizenzinformation auf http://creativecommons.org/licenses/by/4. $0 /$ deed.de.

\section{Literatur}

1. Craig JP, et al. TFOS DEWS II Definition and Classification Report. Ocul Surf. 2017;15(3):276-83. Jul.

2. Clayton JA, et al. Dry Eye. N Engl J Med. 2018;7;378(23): 2212-23. Jun.

3. Shigeyasu C et al. Quality of Life Measures and Health Utility Values Among Dry Eye Subgroups. Health Qual Life Outcomes. 2018;16(1):170. https://doi.org/10.1186/ s12955-018-0999-3

4. Stapleton F, et al. TFOS DEWS II Epidemiology Report. Ocul Surf. 2017;15(3):334-65. Jul.

5. Barabino S, et al. Understanding Symptoms and Quality of Life in Patients With Dry Eye Syndrome. Ocul Surf. 2016;14(3):365-76. Jul.

6. Mishima S. Some physiological aspects of the precorneal tear film. Arch Ophthalmol. 1965;73:233-41.

7. Albarran C, Pons AM, Lorente A, et al. Influence of the tear film on optical quality of the eye. Cont Lens Anterior Eye. 1997;20:129-35.

8. Craig JP, Tomlinson A. Importance of the lipid layer in human tear film stability and evaporation. Optom Vis Sci. 1997;74:8-13.

9. Johnson ME, Murphy PJ. Changes in the tear film and ocular surface from dry eye syndrome. Prog Retin Eye Res. 2004;23:449-74.

10. GotoE, YagiY, MatsumotoY, etal.Impairedfunctional visual acuity ofdryeyepatients. AmJOphthalmol.2002;133:181-6.

11. Chan TCY, et al. Advances in dry eye imaging: the present and beyond. BrJOphthalmol.2018;102(3):295-301. Mar.

12. Tsubota K, etal. New Perspectives on DryEye Definition and Diagnosis: A Consensus Report by the Asia Dry Eye Society. Asia DryEye Society. Ocul Surf. 2017;15(1):65-76.Jan.

13. Nichols KK, Foulks GN, Bron AJ, et al. The international workshop on meibomian gland dysfunction: executive summary. Invest Ophthalmol Vis Sci. 2011;52:1922-9.

14. Foulks GN, Bron AJ. Meibomian gland dysfunction: a clinical scheme for description, diagnosis, classification, and grading. Ocul Surf. 2003;1:107-26.

15. Dry Eye Workshop Committee. 2017 report of the international dry eye workshop (DEWSII). Ocul Surf. 2017;15(3):276-83.

16. Eom Y, Lee JS, Kang SY, et al. Correlation between quantitative measurements of tear film lipid layer thickness and meibomian gland loss in patients with obstructive meibomian gland dysfunction and normal controls. Am J Ophthalmol.2013;155:1104-10.

17. Graudenz K, et al. Diffundierte Schmutz- und Schmucktätowierungen - Entfernung durch gütegeschaltete Laser. Hautarzt. 2003;54:756-9.

18. Adam K Rudkin et al. Wake up with make-up: complication of cosmetic lid tattoo. MJA2011. Volume 194 Number 12.

19. Haddad C. Delayed Complication from a Tattoo. Am Fam Physician. 2012;86:669-70.

20. Messmer EM, et al.Lidline Tattoo Associated with Basal Cell Carcinoma of the Lid Margin -Coincidence or Association? Klin Monatsbl Augenheilkd.2018:785-788.

21. Huisman S, et al. Granulomatous tattoo reactions in permanent makeup of the eyebrows. Cosmet Dermatol. 2019;18(1):212-4. Feb.

22. Goldman A, et al. Severe unexpected adverse effects after permanent eye makeup and their management by Q-switched Nd:YAG laser. Clin Interv Aging. 2014;9:1305-9.

23. Hurwitz JJ, Brownstein S, Mishkin SK. Histopathological findings in blepharopigmentation (eyelid tattoo). Can J Ophthalmol. 1988;23:267-9. 
24. Bourra H, Hassam B. Contact dermatitis after black henna tattoo [inFrench. Pan Afr Med J. 2013;14:154.

25. Turan H, Okur M, Kaya E, et al. Allergic contact dermatitis to paraphenylenediamine in a tattoo: a case report. Cultan Ocul. Toxicol.2013;32:185-97.

26. Bron AK, Tiffany JM. The meibomian glands and tear film lipids: structure, function and control. Adv Exp Med Biol. 1998;438:281-95.

27. Matsumoto Y, Sato EA, Ibrahim OM, et al. The application of in vivo laser confocal microscopy to the diagnosis and evaluation of meibomian gland dysfunction. Mol Vis. 2008;14:1263-71.

28. McCulley JP, Shine WE. Meibomian gland function and the tear lipid layer. Ocul Surf. 2003;1:97-106.

29. Bron AJ, et al. TFOS DEWS II Pathophysiology Report. Ocul Surf. 2017;15(3):438-510. Jul.

30. Laux P, Tralau T, Tentschert J, et al. A medical-toxicological view of tattooing. Lancet. 2016;387:395-402.

31. Islam PS, Chang C, Selmi C, et al. Medical complications of tattoos. A comprehensive review. Clin Rev Allerg Immunol. 2016;50:273-86.
32. Fujiita H, Nishii Y, Yamashita K, et al. The uptake and long-termstorage of India ink particles and latex beads by fibroblasts in the dermis and subcutis of mice, with special regard to the non-inflammatory defense reaction by fibroblasts. Arch Histol Cytol. 1988;51:285-94.

33. Thum CK, Biswas A. Inflammatory complications related to tattooing: a histopathological approach based on pattern analysis. Am J Dermatopathol. 2015;37:54-66.

34. Messmer EM, et al. Lidstrichtattoo assoziiert mit Lidkantenbasaliom - Zufall oder Assoziation? Klin Monbl Augenheilkd.2018;235(7):785-8. Jul.

35. SerupJ, etal.From Technique of Tattooingto Biokinetics and Toxicology of Injected Tattoo Ink Particles and Chemicals. Curr Probl Dermatol.2017;52:1-17.

36. Giulbudagian M, et al. Safety of tattoos and permanent make-up: a regulatory view. Arch Toxicol. 2020;94(2):357-69. Feb.

Hinweis des Verlags Der Verlag bleibt in Hinblick auf geografische Zuordnungen und Gebietsbezeichnungen in veröffentlichten Karten und Institutsadressen neutral. 